\title{
Transtorno de Estresse Pós-Traumático (TEPT) em Bombeiros de Belo Horizonte, Brasil: Prevalência e Fatores Ocupacionais Associados
}

\author{
Eduardo de Paula Lima ${ }^{1}$ \\ Ada Ávila Assunção \\ Sandhi Maria Barreto \\ Universidade Federal de Minas Gerais
}

\begin{abstract}
RESUMO - O objetivo do presente estudo foi estimar a prevalência (últimos 30 dias) de Transtorno de Estresse Pós-Traumático (TEPT) e investigar se variáveis ocupacionais estão associadas ao desfecho em bombeiros de Belo Horizonte, Brasil. Estudo transversal foi realizado em 2011. Dentre 794 elegíveis, 711 (89,5\%) participaram. Informações foram obtidas por questionário de autorrelato: variáveis sociodemográficas e ocupacionais, saúde e eventos extralaborais adversos. A prevalência de TEPT foi $6,9 \%$. Variáveis ocupacionais contribuíram para explicar o desfecho no modelo final (regressão logística multivariável): fatores psicosssociais do trabalho, eventos traumáticos ocupacionais, tempo de trabalho e absenteísmo. Idade, problemas de saúde mental no passado e eventos adversos extralaborais também foram associados ao TEPT. Os resultados são discutidos considerando os pressupostos do Modelo Demanda-Controle.
\end{abstract}

Palavras-chave: bombeiros, prevalência, transtorno de estresse pós-traumático, estressores ocupacionais

\section{Posttraumatic Stress Disorder (PTSD) among Firefighters from Belo Horizonte City, Brazil: Prevalence and Occupational Associated Factors}

\begin{abstract}
The aim of this study was to estimate the prevalence of PTSD (last 30 days) and to investigate whether occupational variables are associated with the outcome in firefighters from Belo Horizonte city, Brazil. The cross-sectional study was conducted in 2011. Among 794 eligible workers, 711 (89.5\%) participated. Information was obtained by self-report questionnaire: sociodemographic and occupational variables, health status and non-occupational adverse events. The prevalence of PTSD was $6.9 \%$. Occupational variables contributed to explain the outcome in the final model (multivariate logistic regression): psychosocial work factors, occupational traumatic events, duration of work as firefighter, and absenteeism. Age, mental health problems in the past, and non-occupational adverse events were also associated with the outcome. The results are discussed considering the assumptions of Demand-Control Model.
\end{abstract}

Keywords: firefighters; prevalence; posttraumatic stress disorder; occupational stressors.

O Transtorno de Estresse Pós-Traumático (TEPT) é uma morbidade relacionada a exposição direta ou indireta a eventos traumáticos como morte, lesões ou traumas graves. Os 20 sintomas típicos descritos no DSM-5 (American Psychiatry Association, 2013) são classificados de acordo com quatro dimensões: revivescência, esquiva, alterações negativas na cognição/humor e excitabilidade aumentada. Para o diagnóstico, é aconselhável perguntar sobre a duração dos sintomas (mínimo de 30 dias) e tentar identificar possíveis prejuízos no funcionamento psicossocial.

Não há dúvidas sobre o peso dos determinantes sociais na prevalência de TEPT (Anakwenze \& Zuberi, 2013). Indivíduos que residem nas áreas mais pobres são mais vulneráveis, porque tais áreas concentram os casos de assaltos, agressões físicas e morte por causas externas (World Health Organization, 2010). No âmbito ocupacional, destaca-se a natureza das tarefas, em especial aquelas desenvolvidas por profissionais de emergências: socorristas em ambulâncias que

1 Endereço para correspondência: Av. Alfredo Balena, ${ }^{\circ}{ }^{\circ} 190$, sala 705, Belo Horizonte, Minas Gerais, Brasil. CEP: 30.310100.E-mail:edpl@hotmail.com prestam atendimento pré-hospitalar, policiais que combatem o crime, e bombeiros que realizam atividades de busca e resgate (de Boer, van'tVerlaat, Duivenvoorden, Bakker, \& Smit, 2011). Esses profissionais lidam cotidianamente com eventos traumáticos e, por esse motivo, estão sob maior risco de adoecer (American Psychiatry Association, 2013).

Ademais, as tarefas desenvolvidas em serviços de emergências coincidem com a exposição a fatores psicossociais negativos (Regehr \& Millar, 2007). Dentre eles, citam-se a alta demanda (necessidade de respostas rápidas) e o baixo controle sobre o trabalho (protocolos pouco ou nada flexíveis), pois a eficácia dos atendimentos depende de ações imediatas e integradas, domínio de conhecimento técnico e emprego adequado dos recursos tecnológicos disponíveis (Adriaenssens, de Gucht, van der Doef, \& Maes, 2012; Carey, Al-Zaiti, Dean, Sessanna, \& Finnell, 2011).

A influência de fatores psicossociais sobre a saúde de trabalhadores é frequentemente abordada sob o enfoque do modelo Demanda-Controle (DC) (Araújo, 2010; U'Ren, 2011). Desenvolvido por Karasek e Theörell (1990), o modelo DC pressupõe que uma alta demanda de trabalho (pressão temporal, jornada extensa, múltiplas tarefas, às 
vezes conflitantes) combinada a um baixo controle sobre o desenvolvimento das tarefas (poucas oportunidades para usar e desenvolver habilidades e baixo poder decisório) caracterizam uma condição de alta exigência psicológica. Tal condição está na origem de (ou pode agravar) vários sintomas mórbidos.

Restrições à autonomia teriam peso sobre o desenvolvimento dos sintomas, pois perturbam o alívio das tensões (arousal) provocadas pelas demandas de trabalho e acarretam limitações para o uso de estratégias de enfrentamento (coping) tanto individuais quanto coletivas.

A terceira dimensão psicossocial de acordo com o modelo DC é o apoio social. Em situações de fraco apoio, é possível que o sujeito se sinta sobrecarregado devido ao excesso de responsabilidade ao ter de enfrentar sozinho as vicissitudes do meio (situações de desespero dos cidadãos acolhidos, por exemplo).

No caso do TEPT em profissionais de emergências, o adoecimento seria resultado da sobreposição dos efeitos: exposição crônica a eventos traumáticos e a fatores psicossociais negativos (Regehr \& Millar, 2007). Em tais situações, as respostas psicofisiológicas extremas (extreme arousal) interfeririam tanto no desenvolvimento quanto na mobilização das estratégias de enfrentamento elaboradas pelos sujeitos (Karasek \& Theörell, 1990).

Investigações anteriores corroboram tais hipóteses, indicando que ambos os grupos de estressores citados (eventos traumáticos e fatores psicossociais negativos) influenciam a ocorrência de TEPT (Berger et al., 2011; Lima \& Assunção, 2011). Fraco apoio social (dos colegas e dos chefes), por exemplo, foi associado ao desfecho em bombeiros japoneses (Mitani, 2008). Bombeiros de Taiwan (Chen et al., 2007) expostos a eventos traumáticos ocupacionais, carga de trabalho elevada e críticas excessivas dos superiores e colegas apresentaram maior chance de adoecer. Em grupos de bombeiros americanos e de bombeiros holandeses, identificou-se padrão similar de associação com eventos traumáticos (Del Ben, Scotti, Chen, \& Fortson, 2006; van der Velden et al., 2006).

O presente estudo busca ampliar a discussão sobre TEPT na medida em que aborda os estressores ocupacionais em uma categoria específica de profissionais de emergências (bombeiros), sob o enfoque do modelo DC. O objetivo foi estimar a prevalência de TEPT em bombeiros de Belo Horizonte e investigar se estressores ocupacionais estavam associados ao desfecho. Espera-se que a exposição a eventos traumáticos ocupacionais e a fatores psicossociais negativos (alta exigência) aumente a chance de TEPT de forma independente na amostra estudada.

Para a investigação da hipótese principal, características sociodemográficas, informações sobre o emprego, saúde geral e exposição a eventos adversos de vida foram considerados possíveis fatores de confusão, pois se mostraram associados a estressores ocupacionais e ao desfecho na população geral (Brewin, Andrews, \& Valentine, 2000; Nieuwenhuijsen, Bruinvels, \& Frings-Dresen, 2010; Ozer, Best, Lipsey, \& Weiss, 2003) e em profissionais de emergências (Carey et al., 2011; Lima \& Assunção, 2011).

\section{Método}

\section{Participantes}

Estudo transversal de base censitária foi realizado em 2011 (fevereiro a agosto). Todos os bombeiros operacionais do Corpo de Bombeiros Militar de Minas Gerais (CBMMG) alocados em Belo Horizonte e com pelo menos 12 meses de trabalho foram convidados a participar. No Brasil, os Corpos de Bombeiros são instituições militares estruturadas sob fortes disciplina e hierarquia, desempenhando atividades de combate a incêndio, resgate, salvamento e atendimento pré-hospitalar. No presente estudo, os bombeiros elegíveis desempenhavam todas as atividades citadas.

O universo dos sujeitos foi identificado nas listas elaboradas pela seção de recursos humanos de cada batalhão. Foram considerados inelegíveis os bombeiros do sexo feminino $(n=70)$, em férias-prêmio ou licença médica durante o período de coleta de dados $(n=30)$, cedidos para outras unidades $(n=30)$ e aqueles que participaram da etapa piloto $(n=30)$ do presente estudo. Uma taxa de resposta mínima de $80 \%$ para cada batalhão e subunidade foi estipulada como critério de inclusão nas análises.

O contingente de mulheres é mínimo $(N=70)$ no universo $(N=954)$ dos trabalhadores do serviço operacional do CBMMG, sendo excluído das análises porque o número, seguindo o preconizado pela literatura (Hair, Anderson, Tatham, \& Black, 2005), inviabilizou a construção de modelos multivariados separados por sexo. A possibilidade de analisar todos os participantes em um mesmo modelo também foi descartada, pois as mulheres são mais susceptíveis ao desenvolvimento de sintomas de TEPT (Blein, Galovski, \& Robinson, 2010), além de elas atuarem menos frequentemente no serviço operacional (setor no qual se dá a exposição aos eventos traumáticos). Ambas as condições criariam um viés de seleção, caso o contingente de mulheres tivesse sido incluído na amostra.

\section{Instrumentos}

As informações foram obtidas por questionário estruturado, cuja adequação e aplicabilidade foram testadas na etapa piloto. Todos os participantes responderam ao questionário no local de trabalho, durante o horário de serviço.

A avaliação de sintomas de TEPT deu-se por meio da versão em português da Posttraumatic Stress Disorder Checklist (PCL-C). A PCL-C é uma escala estruturada de autorrelato composta por 17 itens congruentes com os sintomas de TEPT (Weathers, Litz, Herman, Huska, \& Keane, 1993). O respondente indica o quanto ele foi incomodado no último mês pelos sintomas descritos, utilizando uma escala Likert de intensidade que varia de um (nada) a cinco (muito). $\mathrm{O}$ escore final pode variar de 17 a 85 pontos. $\mathrm{O}$ escore total maior ou igual a 50 pontos é considerado ponto de corte ótimo para casos prováveis de TEPT (Weathers et al., 1993), sendo que, no presente estudo, foi utilizado para estimar a prevalência nos últimos 30 dias. A versão semanticamente 
equivalente da PCL-C no Brasil foi desenvolvida em 2004 (Berger, Mendlowicz, Souza, \& Figueira, 2004). Estudos sobre as características psicométricas da versão em português indicaram índices satisfatórios de validade fatorial, confiabilidade temporal e consistência interna (Lima, Barreto, \& Assunção, 2012).

A exposição a eventos traumáticos ocupacionais foi avaliada pela Posttraumatic Diagnostic Scale adaptada para profissionais de emergências (PDS-PE) (Laposa \& Alden, 2003). O instrumento consiste em uma lista de 15 eventos potencialmente traumáticos típicos em serviços de emergências, sendo utilizado em substituição à lista de eventos gerais apresentados na versão original da escala (Foa, Cashman, Jaycox, \& Perry, 1997). O respondente deve indicar quantos eventos vivenciou durante o trabalho nos últimos 12 meses e qual deles mais o incomodou.

Para o presente estudo, realizou-se a tradução e análise semântica da PDS-PE para profissionais de emergências em cinco etapas, incluindo tradução e tradução reversa, apreciação formal de equivalência, interlocução com profissionais de emergências em hospitais, policiais civis e policiais militares e investigação das características psicométricas. $\mathrm{O}$ escore total na escala pode variar de 0 a 45 pontos. A PDS-PE foi incluída nas análises como variável dicotômica, considerando o $5^{\circ}$ quintil na escala como ponto de corte.

Dois tipos de estressores organizacionais foram avaliados: fatores psicossociais do trabalho e condições do ambiente de trabalho. O Job Content Questionnaire (JCQ) adaptado para o português por Araújo e Karasek (2008) foi utilizado para avaliar demanda física e psicológica, controle sobre o trabalho e apoio social. O instrumento foi construído para operacionalizar o Modelo Demanda-Controle (Karasek \& Theörell, 1990). As dimensões do JCQ foram transformadas em variáveis dicotômicas por meio da mediana. Na sequência, demanda física e psicológica e controle sobre as tarefas foram combinados em quatro situações de trabalho: 1) alta exigência (alta demanda e baixo controle); 2) trabalho ativo (alta demanda e alto controle); 3) trabalho passivo (baixa demanda e baixo controle); e 4) trabalho de baixa exigência (baixa demanda e alto controle) (Karasek \& Theörell, 1990). O apoio social foi analisado separadamente como variável dicotômica.

Condições de trabalho foram abordadas por meio de perguntas sobre disponibilidade de equipamentos de proteção individual, ruído no local de trabalho e aquele originado fora do local de trabalho e disponibilidade de recursos materiais suficientes para executar as tarefas. As respostas aos itens mencionados foram somadas para a construção de um escore composto e analisadas como variável ordinal.

Possíveis fatores de confusão também foram considerados nas análises: características sociodemográficas (idade, estado civil, número de filhos, escolaridade, raça/cor da pele autodeclarada e renda mensal), informações sobre o emprego (tempo de trabalho, absenteísmo e posição hierárquica), saúde e eventos adversos de vida.

Questões sobre saúde incluíram: 1) transtornos mentais no passado (tratamento psicológico e/ou psiquiátrico, uso de psicotrópicos ou diagnóstico médico de transtornos psiquiátricos nos últimos doze meses). Considerou- se presença de transtornos mentais a afirmação pelo respondente de pelo menos um dos eventos interrogados; 2) diagnóstico de doenças crônicas (hipertensão, diabetes, asma/bronquite, infarto do miocárdio, enfisema e distúrbios musculoesqueléticos). Aqueles participantes que indicaram pelo menos um dos diagnósticos listados no questionário foram considerados como portadores de doenças crônicas; 3) prática de atividades físicas; 4) tabagismo; e 5) uso problemático de álcool.

O uso problemático de álcool foi investigado utilizando o CAGE (Masur \& Monteiro, 1983) e não foi restrito ao último ano. Suas quatro questões são identificadas pelas palavraschave: Cutdown (C); Annoyed (A); Guilty (G); e Eye-opener (E). O CAGE foi analisado como uma variável dicotômica (duas ou mais respostas afirmativas no questionário indicaram histórico de uso problemático de álcool).

Por fim, a vivência de eventos adversos extralaborais nos últimos 12 meses foi avaliada por meio de questões referentes a dois domínios: eventos estressantes e eventos traumáticos. Os eventos estressantes foram os seguintes: problemas gerais de saúde, problemas financeiros graves, abandono involuntário de residência, falecimento de familiar, rompimento de relacionamento amoroso e exposição à discriminação social (raça, gênero, orientação sexual, religião, deficiência física, idade e condição socioeconômica). Os eventos traumáticos foram listados em consonância com o Critério A descrito no DSM e incluíram: internação hospitalar por problema grave de saúde ou acidente, assalto e agressão física. As vivências de situações extralaborais foram analisadas como variáveis ordinais.

\section{Procedimentos}

Os participantes de posse do termo de consentimento livre e esclarecido foram informados quanto aos objetivos e ao caráter confidencial e voluntário da pesquisa. O projeto foi aprovado pelo CBMMG e pelo Comitê de Ética em Pesquisa da Universidade Federal de Minas Gerais (ETIC no 0387.0.203.000-10).

\section{Análise}

As análises foram realizadas no SPSS, versão 17.0, em quatro etapas: 1) análise descritiva, incluindo prevalência pontual nos últimos 30 dias; 2) análise univariável (regressão logística simples) para verificar os fatores associados aos prováveis casos de TEPT $(p<0,20) ; 3)$ análise multivariável por blocos (regressão logística múltipla) incluindo todas as variáveis associadas aos casos prováveis de TEPT na análise univariável. Quatro blocos foram construídos (variáveis sociodemográficas e hábitos de vida; estressores ocupacionais e informações sobre o emprego, saúde, e eventos adversos de vida); 4) análise multivariável (regressão logística múltipla), incluindo todas as variáveis associadas aos casos prováveis de TEPT $(p<0,10)$ na análise multivariável por bloco. Apenas as variáveis associadas ao nível de significância igual ou menor a $5 \%(p<0,05)$ permaneceram no modelo final. 
Quanto ao método de análise, ressalta-se que diferentes modelos de regressão logística foram testados, incluindo a regressão hierárquica e a estatística. Os resultados foram consistentes independentemente da estratégia adotada. Optou-se por utilizar a regressão estatística. A regressão estatística seria adequada ao intuito de produzir resultados de caráter exploratório, pois convém assumir que ainda são raros os estudos que fazem referência ao modelo DemandaControle quando avaliam os fatores psicossociais do trabalho na explicação de casos prováveis de TEPT. Diante da carência de estudos com tal referencial, seria precipitado, como alerta Abbad e Torres (2002), adotar uma abordagem hierárquica. A nosso ver, a regressão estatística (stepwise por blocos de variáveis) é a mais adequada para investigar as hipóteses aventadas, sendo que tal opção encontra apoio na literatura (Tabachnick \& Fidell, 2013).

\section{Resultados}

De um total de 954 bombeiros alocados em unidades operacionais de Belo Horizonte, 794 sujeitos foram considerados elegíveis, tendo sido obtida a participação de 711 (89,5\% dos elegíveis). O resultado da coleta em todos os batalhões e subunidades atingiu a meta, variando de $80,0 \%$ a $96,9 \%$ a taxa de resposta. A comparação entre respondentes e não-respondentes indicou que os dois grupos não diferiram em relação à idade $(p=0,106)$, escolaridade $(p=309)$, estado civil $(p=0,677)$, posto hierárquico $(p=0,113)$, unidade de trabalho $(p=0,218)$ e tempo de serviço no CBMMG ( $p$ $=0,117$ )

O escore médio dos participantes na PCL-C foi 29,3 $(d p=11,8)$ e a prevalência de casos prováveis de TEPT nos últimos 30 dias foi de 6,9\% (IC 95\%= 5,2\%-9,0\%). Observou-se predomínio dos participantes entre 30 e 39 anos (31,9\%). A maioria declarou-se casada ou em união estável $(55,4 \%)$, nível fundamental ou secundário de escolaridade $(66,0 \%)$, com filhos $(53,1 \%)$, cor da pele preta ou parda $(65,5 \%)$. Quanto à renda familiar, 41,6\% declararam rendimento mensal entre $\mathrm{R} \$ 2.501,00$ e $\mathrm{R} \$ 4.000,00$. A análise univariável indicou que bombeiros mais jovens (entre 19 e 24 anos de idade), solteiros e sem filhos apresentaram menores prevalências de TEPT (vide Tabela 1).

Cerca $36,0 \%$ dos participantes estavam trabalhando no setor há menos de três anos. Predominaram aqueles na graduação de "soldado" $(45,3 \%)$, sem relato de absenteísmo nos últimos 12 meses $(56,0 \%)$. Quanto à exposição a estressores ocupacionais, a maior parte dos participantes relatou duas ou mais condições precárias no ambiente de trabalho $(50,4 \%) ; 27,3 \%$ percebem alta demanda e baixo controle no trabalho, caracterizando uma situação de alta exigência. A análise univariável indicou que bombeiros no

Tabela 1. Características sociodemográficas e casos prováveis de TEPT em bombeiros de Belo Horizonte, Brasil.

\begin{tabular}{|c|c|c|c|c|}
\hline \multirow{2}{*}{ Variáveis } & \multirow{2}{*}{ Categorias } & \multicolumn{2}{|c|}{ TEPT } & \multirow{2}{*}{ OR $(I C 95 \%)$} \\
\hline & & $\operatorname{Sim} N(\%)$ & Não $N(\%)$ & \\
\hline \multirow[t]{4}{*}{ 1.Idade (anos) } & $19-24$ & $4(2,5 \%)$ & $155(97,5 \%)$ & - \\
\hline & $25-29$ & $12(9,7 \%)$ & $112(90,3 \%)$ & $4,15(1,31-13,21)^{* *}$ \\
\hline & $30-39$ & $16(7,3 \%)$ & $203(92,7 \%)$ & $3,05(1,00-9,32)^{* *}$ \\
\hline & $40-50$ & $16(8,5 \%)$ & $172(91,5 \%)$ & $3,60(1,18-11,01)^{* *}$ \\
\hline \multirow[t]{3}{*}{ 2. Escolaridade } & Ensino fundamental & $3(5,7 \%)$ & $50(94,3 \%)$ & - \\
\hline & Ensinomédio & $29(6,4 \%)$ & $424(93,6 \%)$ & $1,14(0,33-3,88)$ \\
\hline & Gradução/Pós-Graduação & $16(8,7 \%)$ & $168(91,3 \%)$ & $1,59(0,44-5,69)$ \\
\hline \multirow[t]{3}{*}{ 3.Estado Civil } & Casado/Uniãoestável & $29(7,7 \%)$ & $348(92,3 \%)$ & - \\
\hline & Solteiro & $16(5,7 \%)$ & $267(94,3 \%)$ & $0,72(0,38-1,35)$ \\
\hline & Separado/Divorciado & $3(9,4 \%)$ & $29(90,6 \%)$ & $1,24(0,36-4,32)$ \\
\hline \multirow[t]{2}{*}{ 4. Filhos } & Não & $18(5,5 \%)$ & $312(94,5 \%)$ & - \\
\hline & Sim & $30(8,3 \%)$ & $331(91,7 \%)$ & $1,57(0,85-2,88)^{*}$ \\
\hline \multirow[t]{4}{*}{ 5. Raça / Cor da pele } & Branca & $14(6,7 \%)$ & $196(93,3 \%)$ & - \\
\hline & Parda & $27(7,5 \%)$ & $331(92,5 \%)$ & $1,14(0,58-2,23)$ \\
\hline & Preta & $5(5,4 \%)$ & $88(94,6 \%)$ & $0,80(0,28-2,28)$ \\
\hline & Asiática / Indígena & $2(7,1 \%)$ & $26(92,9 \%)$ & $1,08(0,23-5,01)$ \\
\hline \multirow[t]{5}{*}{ 6. Renda } & $<\mathrm{R} \$ 2.500$ & $13(7,8 \%)$ & $153(92,2 \%)$ & - \\
\hline & $\mathrm{R} \$ 2.501-\mathrm{R} \$ 4.000$ & $20(7,0 \%)$ & $266(93,0 \%)$ & $0,88(0,42-1,83)$ \\
\hline & $\mathrm{R} \$ 4.001-\mathrm{R} \$ 5.500$ & $8(6,3 \%)$ & $119(93,7 \%)$ & $0,79(0,31-1,97)$ \\
\hline & $\mathrm{R} \$ 5.501-\mathrm{R} \$ 7.000$ & $6(9,7 \%)$ & $56(90,3 \%)$ & $1,26(0,46-3,48)$ \\
\hline & $>\mathrm{R} \$ 7.000$ & $1(2,0 \%)$ & $50(98,0 \%)$ & $0,24(0,03-1,84)^{*}$ \\
\hline
\end{tabular}

Nota: * Variáveis associadas a casos prováveis de TEPT (PCL-C $>50$ na análise univariável $(p<0,20)$; ** Variáveis associadas a casos prováveis de TEPT (PCL-C $>50)$ na análise univariável $(p<0,05)$. 
posto de "cabo", com três ou mais anos de serviço e expostos à baixo apoio social, eventos traumáticos ocupacionais e situação de alta exigência no trabalho apresentaram maiores prevalências de TEPT (Tabela 2).

Quanto à saúde, 20,7\% dos bombeiros informaram diagnóstico clínico para alguma doença crônica e 16\% relataram problemas de saúde mental no passado. Os respondentes são predominantemente não fumantes $(82,6 \%)$ e praticam atividade física uma a duas vezes por semana $(45,1 \%)$. A avaliação da vivência de eventos extralaborais adversos revelou que a maior parte dos participantes foi exposta a eventos adversos de vida no último ano, incluindo situações traumáticas $(14,4 \%)$, estressantes $(59,5 \%)$ ou preconceito social (25,0\%) (Tabela 3). À análise univariável, viu-se maior prevalência de TEPT no grupo que informou uso abusivo de álcool, diagnóstico de uma ou mais doenças crônicas, transtorno mental no passado, pouca ou nenhuma atividade física e vivência de eventos extralaborais adversos (traumáticos, estressantes e discriminação social) (vide Tabela 2).

Análises multivariáveis separadas por blocos mantiveram as seguintes variáveis: 1) idade; 2) estressores ocupacionais (trabalho de alta exigência e eventos traumáticos no trabalho), posto hierárquico, absenteísmo e tempo de serviço; 3) uso abusivo de álcool, doenças crônicas e transtornos mentais no passado; 4) vivência de eventos extralaborais adversos (traumáticos e estressantes).

O modelo multivariável final manteve as seguintes variáveis: idade, exposição a estressores ocupacionais (trabalho de alta exigência e eventos traumáticos), absenteísmo, tempo de trabalho no setor, transtornos mentais no passado e eventos extralaborais estressantes (vide Tabela 4).

\section{Discussão}

A prevalência de casos prováveis de TEPT (últimos 30 dias) em bombeiros de Belo Horizonte foi similar às taxas encontradas em outros grupos de profissionais de emergências e superior à encontrada na população geral. Associações significativas foram identificadas entre eventos traumáticos ocupacionais, fatores psicossociais do trabalho (alta exigência), absenteísmo, tempo de serviço e TEPT (casos prováveis). No conjunto, os resultados sugerem a relevância das variáveis ocupacionais na explicação do desfecho.

Os nossos resultados são semelhantes aos obtidos em investigações nos Estados Unidos (EUA), cujas prevalências variaram entre 4,2\% (Meyer et al., 2012) e 6,0\% (Del Ben et al., 2006). Contudo, foram encontradas taxas superiores no

Tabela 2. Variáveis ocupacionais e casos prováveis de TEPT em bombeiros de Belo Horizonte, Brasil

\begin{tabular}{|c|c|c|c|c|}
\hline \multirow{2}{*}{ Variáveis } & \multirow{2}{*}{ Categorias } & \multicolumn{2}{|c|}{ TEPT } & \multirow{2}{*}{ OR $(I C \mathbf{9 5 \%})$} \\
\hline & & $\operatorname{Sim} N(\%)$ & Não $N(\%)$ & \\
\hline \multicolumn{5}{|l|}{ 1. Fatores psicossociais - JCQ } \\
\hline Baixa exigência & $7(5,9 \%)$ & $111(94,1 \%)$ & - & \\
\hline Trabalho passivo & $7(4,1 \%)$ & $165(95,9 \%)$ & $0,67(0,23-1,97)$ & \\
\hline Trabalho ativo & $9(4,4 \%)$ & $195(95,6 \%)$ & $0,73(0,27-2,02)$ & \\
\hline Alta exigência & $24(13,0 \%)$ & $161(87,0 \%)$ & $2,36(0,99-5,68)^{*}$ & \\
\hline \multirow[t]{2}{*}{ Apoio Social } & Alto & $23(8,9 \%)$ & $236(91,1 \%)$ & - \\
\hline & Baixo & $25(5,8 \%)$ & $408(94,2 \%)$ & $1,59(0,88-2,87)^{*}$ \\
\hline \multirow[t]{2}{*}{ 2. Eventos traumáticos - PDS } & $<$ 5oquintil & $28(5,2 \%)$ & $515(94,8 \%)$ & - \\
\hline & $>$ 5oquintil & $20(15,5 \%)$ & $109(84,5 \%)$ & $3,37(1,83-6,21)^{* *}$ \\
\hline \multirow[t]{2}{*}{ 3. Absenteísmo } & Não & $8(2,1 \%)$ & $377(97,9 \%)$ & - \\
\hline & Sim & $38(12,8 \%)$ & $259(87,2 \%)$ & $6,91(3,17-15,06)$ \\
\hline \multirow[t]{3}{*}{ 4. Ambiente de trabalho (número de condições precárias) } & 0 & $4(5,1 \%)$ & $75(94,9 \%)$ & - \\
\hline & 1 & $11(4,2 \%)$ & $251(95,8 \%)$ & $0,82(0,25-2,66)$ \\
\hline & 2 ou mais & $33(9,5 \%)$ & $314(90,5 \%)$ & $1,97(0,68-5,73)$ \\
\hline \multirow[t]{3}{*}{ 5. Tempo de trabalho no CBMMG (anos) } & $<3$ & $7(2,8 \%)$ & $245(97,2 \%)$ & - \\
\hline & $3-16$ & $20(9,1 \%)$ & $200(90,9 \%)$ & $3,5(1,45-8,44)^{* *}$ \\
\hline & $17-30$ & $21(10,1 \%)$ & $186(89,9 \%)$ & $3,95(1,65-9,50)^{* *}$ \\
\hline \multirow[t]{4}{*}{ 6.Posto } & Soldado & $17(5,3 \%)$ & $304(94,7 \%)$ & - \\
\hline & Cabo & $15(10,6 \%)$ & $126(89,4 \%)$ & $2,13(1,03-4,39)^{* *}$ \\
\hline & Sargento & $12(6,6 \%)$ & $171(93,4 \%)$ & $1,25(0,58-2,69)$ \\
\hline & Oficial & $4(8,5 \%)$ & $43(91,5 \%)$ & $1,66(0,53-5,18)$ \\
\hline
\end{tabular}

Nota: * Variáveis associadas a casos prováveis de TEPT (PCL-C $>50)$ na análise univariável $(p<0,20)$; ** Variáveis associadas a casos prováveis de TEPT (PCL-C $>50)$ na análise univariável $(p<0,05)$. 
Tabela 3. Saúde, eventos extralaboraisadversos e casos prováveis de TEPT em bombeiros de Belo Horizonte, Brasil

\begin{tabular}{|c|c|c|c|c|}
\hline \multirow{2}{*}{ Variáveis } & \multirow{2}{*}{ Categorias } & \multicolumn{2}{|c|}{ TEPT } & \multirow{2}{*}{$O R(I C \mathbf{9 5 \%})$} \\
\hline & & $\operatorname{Sim} N(\%)$ & Não $N(\%)$ & \\
\hline \multirow[t]{2}{*}{ 1. Abuso de álcool } & Não & $33(5,3 \%)$ & $588(94,7 \%)$ & - \\
\hline & Sim & $15(22,1 \%)$ & $53(77,9 \%)$ & $5,04(2,58-9,87)^{* *}$ \\
\hline \multirow[t]{3}{*}{ 2. Doenças crônicas (número) } & 0 & $28(5,2 \%)$ & $516(94,8 \%)$ & - \\
\hline & 1 & $10(8,8 \%)$ & $104(91,2 \%)$ & $1,77(0,84-3,76)^{*}$ \\
\hline & 2 ou mais & $10(35,7 \%)$ & $18(64,3 \%)$ & $\begin{array}{c}10,24(4,33- \\
24,23)^{* *}\end{array}$ \\
\hline \multirow[t]{2}{*}{ 3.Transtornosmentais (passado) } & Não & $19(3,3 \%)$ & $555(96,7 \%)$ & - \\
\hline & Sim & $29(25,7 \%)$ & $84(74,3 \%)$ & $\begin{array}{c}10,08(5,41- \\
18,79)^{* *}\end{array}$ \\
\hline \multirow[t]{3}{*}{ 4. Tabagismo } & Não-fumante & $32(5,6 \%)$ & $538(94,4 \%)$ & - \\
\hline & Ex-fumante & $11(15,9 \%)$ & $58(84,1 \%)$ & $3,19(1,53-6,67)^{* *}$ \\
\hline & Fumante & $5(9,4 \%)$ & $48(90,6 \%)$ & $1,75(0,65-4,70)$ \\
\hline \multirow[t]{3}{*}{ 5. Atividadefísica (frequênciasemanal) } & $>3$ & $12(3,9 \%)$ & $300(96,1 \%)$ & - \\
\hline & 1 ou 2 & $30(8,8 \%)$ & $313(91,2 \%)$ & $2,40(1,20-4,77)^{* *}$ \\
\hline & Nunca & $6(19,4 \%)$ & $25(80,6 \%)$ & $6,00(2,07-17,34)^{* *}$ \\
\hline \multirow[t]{3}{*}{ 6. Eventos traumáticos } & 0 & $30(5,1 \%)$ & $561(94,9 \%)$ & - \\
\hline & 1 & $11(15,5 \%)$ & $60(84,5 \%)$ & $3,43(1,64-7,19)^{* *}$ \\
\hline & 2 ou mais & $7(24,1 \%)$ & $22(75,9 \%)$ & $5,95(2,36-15,03)^{* *}$ \\
\hline \multirow[t]{3}{*}{ 7. Eventos estressantes } & 0 & $4(1,4 \%)$ & $275(98,6 \%)$ & - \\
\hline & 1 & $14(5,9 \%)$ & $225(94,1 \%)$ & $4,28(1,39-13,18)^{* *}$ \\
\hline & 2 ou mais & $30(17,7 \%)$ & $140(82,3 \%)$ & $\begin{array}{c}14,73(5,09- \\
42,65)^{* *}\end{array}$ \\
\hline \multirow[t]{3}{*}{ 8. Preconceito } & 0 & $26(5,0 \%)$ & $490(95,0 \%)$ & - \\
\hline & & $12(10,3 \%)$ & $104(89,7 \%)$ & $2,17(1,06-4,45)^{* *}$ \\
\hline & 2 ou mais & $9(17,0 \%)$ & $44(83,0 \%)$ & $3,86(1,70-8,74)^{* *}$ \\
\hline
\end{tabular}

Nota: * * Variáveis associadas a casos prováveis de TEPT (PCL-C $>50)$ na análise univariável $(p<0,20)$; ** Variáveis associadas a casos prováveis de TEPT (PCL-C $>50)$ na análise univariável $(p<0,05)$.

Japão - 22,0\% (Mitani, 2008) e Taiwan - 10,5\% (Chen et al., 2007). É possível que as prevalências mais altas nos países asiáticos sejam devidas não apenas a diferenças culturais, mas à maior frequência e magnitude de catástrofes "naturais" (Berger et al., 2011).

No Brasil, os estudos no tema evidenciaram menores prevalências no Rio de janeiro (bombeiros) e em São Paulo (população geral), 5,6\% (Berger et al., 2007) e 1,6\% (Andrade et al., 2012), respectivamente. No primeiro caso, a similitude com os nossos resultados perde significado se considerado o critério menos estrito adotado pelos autores para a definição de casos prováveis. Utilizando tal classificação, a prevalência de casos prováveis de TEPT em bombeiros de Belo Horizonte teria sido $10,6 \%$. No caso de São Paulo, a diferença dos resultados pode ser atribuída à técnica de entrevistas clínicas estruturadas. Ademais, os indivíduos inativos e desempregados foram incluídos na amostra, configurando um perfil distinto da amostra dos bombeiros de Belo Horizonte, exclusivamente empregados com garantias de estabilidade e proteção social, ambas condições favoráveis a melhores condições de saúde.

Alta exposição a eventos traumáticos no trabalho foi associada ao desfecho, sendo consistente (Slottje et al., 2008;
Su et al., 2007; van der Velden et al., 2006). Provavelmente, a natureza das atividades desempenhadas pelos bombeiros influencie negativamente a saúde mental, independente de outros estressores ocupacionais (Lima \& Assunção, 2011). Entretanto, ressalta-se que a PCL-C, instrumento utilizado para identificação de casos prováveis de TEPT, é inespecífica, ou seja, não permite identificar qual evento traumático está atrelado aos sintomas. Em consequência, seria precipitado afirmar sobre a origem ocupacional de TEPT na amostra investigada.

Fatores psicossociais do trabalho mostraram-se associados ao TEPT. O modelo DC pressupõe maiores riscos à saúde física e mental em situações de alta exigência (alta demanda e baixo controle) das tarefas (Nieuwenhuijsen, Bruinvels, \& Frings-Dresen, 2010). Os nossos resultados corroboraram tais hipóteses. Tal combinação foi associada à maior chance de casos prováveis de TEPT na amostra estudada.

De acordo com regulamentação interna, o deslocamento dos bombeiros não deve ultrapassar 60 segundos após o registro do alerta nas unidades. Ainda em relação à organização temporal do trabalho nos batalhões, os sujeitos estão expostos a horários atípicos: turnos de 24 horas, em regime de plantão, seguidos de 48 horas de folga, somando 
Tabela 4. Regressão logística multivariável para casos prováveis de TEPT (PCL-C>50) em bombeiros de Belo Horizonte, Brasil

\begin{tabular}{|c|c|c|}
\hline Variáveis & Categorias & $O R(C I \mathbf{9 5 \%})$ \\
\hline \multirow[t]{4}{*}{ 1. Idade (anos) } & $19-24$ & - \\
\hline & $25-29$ & $8,81(1,81-42,86)^{* *}$ \\
\hline & $30-39$ & $1,31(0,24-7,22)$ \\
\hline & $40-50$ & $0,60(0,08-4,55)$ \\
\hline \multirow[t]{4}{*}{ 2. Fatores psicossociais do trabalho (JCQ) } & Baixa exigência & - \\
\hline & Trabalho passivo & $0,69(0,19-2,41)$ \\
\hline & Trabalho ativo & $0,49(0,13-1,76)$ \\
\hline & Alta exigência & $3,21(1,06-9,72)^{*}$ \\
\hline \multirow[t]{2}{*}{ 3. Eventos traumáticos no trabalho (PDS) } & $<5$ oquintil & - \\
\hline & $>5$ o quintil & $2,31(1,02-5,22)^{*}$ \\
\hline \multirow[t]{2}{*}{ 4. Absenteísmo } & Não & - \\
\hline & Sim & $2,94(1,12-7,73)^{*}$ \\
\hline \multirow[t]{3}{*}{ 5. Tempo de trabalho no CBMMG (anos) } & $<3$ & - \\
\hline & $3-16$ & $3,58(1,03-12,77)^{*}$ \\
\hline & $17-30$ & $8,79(1,50-51,55)^{*}$ \\
\hline \multirow[t]{2}{*}{ 6. Transtornosmentais (passado) } & Não & - \\
\hline & Sim & $5,61(2,63-12,00)^{* *}$ \\
\hline \multirow[t]{3}{*}{ 7. Eventos adversos extralaborais (estressantes) } & 0 & - \\
\hline & 1 & $2,62(0,77-8,87)$ \\
\hline & 2 ou mais & $6,85(2,08-22,46)^{* *}$ \\
\hline
\end{tabular}

Nota: *Variáveis associadas a casos prováveis de TEPT (PCL-C $>50)$ na análise multivariável $(p<0,05) ; * *$ Variáveis associadas a casos prováveis de TEPT $(\mathrm{PCL}-\mathrm{C}>50)$ na análise multivariável $(p<0,01)$.

cerca de 56 horas de trabalho semanais (Batista, 2009). No conjunto, a natureza das tarefas e o quadro técnicoorganizacional em que elas se desenvolvem convergem para um contexto de alta demanda e trabalho sob pressão temporal.

De acordo com o modelo DC, a dimensão controle sobre o trabalho implica em dois componentes básicos: 1) aspectos ligados ao desenvolvimento de habilidades, incluindo aprendizagem, criatividade e possibilidade de aperfeiçoamento; e 2) autonomia de decisão, indicando o quanto o indivíduo é capaz de definir seu próprio trabalho (Karasek \& Theörell, 1990). Se, por um lado, os imprevistos oferecem aos bombeiros oportunidades para o desenvolvimento de novas habilidades, a autonomia, por outro lado, parece limitada não apenas pelo arranjo da hierarquia, mas também pela natureza das tarefas que é imperativa na definição das demandas de trabalho.

Quanto ao quadro técnico-organizacional, vale lembrar que o CBMMG é uma instituição militar, cuja hierarquia é rígida, sendo a organização estruturada por códigos de conduta bem definidos e supervisionados. O fluxo de atendimento às ocorrências também porta efeitos nítidos sobre as dimensões controle e demanda das tarefas. O supervisor que recebe as chamadas telefônicas destaca a equipe que vai agir naquele caso de acordo com as viaturas que estão disponíveis na região do evento para "ganhar tempo" (Batista, 2009). Portanto, a racionalidade do atendimento em regime de urgência traz restrições para a autonomia sobre onde, como e quando agir.

Karasek e Thörell (1990) sugerem que a rigidez das formas de controle está relacionada com reações de estresse e inibição do desenvolvimento de estratégias funcionais de enfrentamento (coping). Vale mencionar que os mecanismos sociais, incluindo os contextos ocupacionais, que bloqueiam as estratégias de enfrentamento dos indivíduos, aumentam o risco de adoecimento psíquico (Cyrulnik, 2009).

É possível supor um efeito cumulativo da exposição a estressores ocupacionais sobre a saúde mental. Tal hipótese baseia-se no gradiente dose-resposta observado nas associações entre tempo de serviço e casos prováveis de TEPT: quanto maior a antiguidade no setor, maior a chance de apresentar o desfecho. Esse resultado é convergente com outras investigações sobre profissionais de emergências (Chang et al., 2008; Heinrichs et al., 2005). Ainda no âmbito ocupacional, a relação entre absenteísmo e casos prováveis de TEPT no modelo multivariável final sugere que o grupo com problemas mais graves de saúde (licenciados) tem mais chance de apresentar sintomas compatíveis com o transtorno. Problemas de saúde física e mental que levam ao absenteísmo são frequentemente associados ao TEPT e parecem aumentar a vulnerabilidade a eventos traumáticos. É importante notar que encontramos associação entre histórico de transtornos mentais (de humor e ansiedade) e casos prováveis de TEPT, sendo convergente com a literatura (Chen et al., 2007; van 
der Velden Kleber, Grievink, \& Yzermans, 2010). Contudo, o desenho transversal não exclui a causalidade reversa: o absenteísmo pode ser consequência de sintomas graves de TEPT.

Quanto aos fatores individuais, os resultados indicaram uma associação não-linear entre idade e casos prováveis de TEPT: bombeiros entre 25 e 29 anos de idade apresentaram uma chance maior de apresentar sintomas compatíveis com o transtorno. Ressalta-se que tal associação foi significativa mesmo controlando o efeito do tempo de serviço, enfraquecendo, portanto, a hipótese relacionado ao efeito do trabalhador sadio. As características dos trabalhadores relacionadas à geração talvez tenham influenciado tal resultado (Chau et al., 2010).

Em relação às vivências extralaborais, apenas eventos de vida estressantes (não traumáticos) foram associados ao desfecho. De forma surpreendente, a associação de eventos traumáticos de vida com TEPT (casos prováveis) não foi significativa. Esse resultado seria a manifestação das fraquezas na definição de evento traumático? Há controvérsias quanto a isso, como se viu no processo de elaboração do DSM-5 (Brewin, Andrews, \& Valentine, 2009). O critério A foi revisado mais de uma vez desde a publicação do DSM-III em 1980 e sua definição no DSM-5 (American Psychiatry Association, 2013) foi duramente criticada (Rosen \& Lilienfeld, 2008). O estudo de Gold, Marx, Soler-Baillo e Sloan (2005) focou estudantes universitários expostos a eventos estressantes (não traumáticos). Constatou-se mais chance de sintomas compatíveis com TEPT no referido grupo quando comparado ao grupo exposto a eventos traumáticos congruentes com o critério A do DSM. O resultado sugere que tanto a inconsistência na definição do evento, quanto as lacunas na caracterização dos sintomas estejam contribuindo para as divergências dos resultados (Gold et al., 2005). É possível que os resultados apresentados tenham sido influenciados pelas insuficiências atribuídas ao DSM. Estudos futuros utilizando os novos critérios diagnósticos poderão elucidar a questão.

A presente investigação apresenta contribuições importantes para a explicação do TEPT, reconhecidamente complexo e multideterminado. A alta taxa de participação $(89,5 \%)$ dos bombeiros lotados nas unidades de Belo Horizonte e o uso de instrumentos adaptados para o contexto brasileiro garantiram a validade interna e externa do estudo. A equipe de coleta foi devidamente treinada e supervisionada. A realização de um estudo piloto favoreceu a adequação dos itens construídos e a adesão dos participantes. Em conjunto, os elementos descritos indicam a força do presente estudo e a acurácia dos resultados apresentados.

Ainda assim, algumas limitações devem ser destacadas. O delineamento transversal do presente estudo limita inferências causais na interpretação dos resultados. Não é possível, portanto, descartar a possibilidade de causalidade reversa. Vale destacar que estudos em amostras de trabalhadores ativos sofrem influências do efeito do trabalhador sadio (Silva \& Barreto, 2010). Bombeiros com problemas de saúde, incluindo aqueles com sintomas graves de TEPT, não participaram do inquérito devido a licenças médicas (critério de elegibilidade). Ademais, aposentadoria precoce ou mudança de trabalho por motivo de saúde também podem ter levado a subestimação da prevalência pontual. A exclusão das mulheres limitou o alcance dos resultados. Contudo, se por um lado seria desejável aprofundar os diferenciais de gênero, o desenho do estudo, por outro lado, não permitiria identificar a influência do absenteísmo-doença e susceptibilidade para TEPT que atingem homens e mulheres de forma diferenciada, com desvantagem para as últimas (Blein, Galovski, \& Robinson, 2010).

Quanto ao instrumento, ainda não foi definido o ponto de corte ideal para a versão em português da PCL-C, gerando incertezas nas análises. Serão necessários estudos adicionais relativos à sensibilidade e especificidade da versão disponível.

Alguns fatores associados ao TEPT na população geral não foram avaliados no presente estudo. Experiências traumáticas no passado, abuso físico ou sexual na infância e histórico de problemas psiquiátricos na família podem ser variáveis importantes na explicação de sintomas de TEPT (Brewin, Andrews, \& Valentine, 2000). O corte temporal (últimos 12 meses) na mensuração de eventos traumáticos ocupacionais e vivência de eventos adversos extralaborais pode ter limitado os resultados obtidos. Ademais, traços de personalidade (agressividade e autoeficácia) frequentemente associados à morbidade (Heinrichs et al., 2005) não foram mensurados. Sugere-se a incorporação de tais aspectos em investigações futuras.

\section{Referências}

Abbad, G., \& Torres, C. V. (2002) Regressão múltipla stepwise e hierárquica em Psicologia Organizacional: aplicações, problemas e soluções. Estudos de Psicologia, 7(1),19-29.

Andrade, L. H., Wang, Y. P., Andreoni, S., Silveira, C. M., Alexandrino-Silva, C., Siu, E. R.,...Viana, M. C. (2012). Mental Disorders in Megacities: Findings from the São Paulo Megacity Mental Health Survey, Brazil. Plos One, 7(2), e31879. doi: doi:10.1371/journal.pone.0031879

Adriaenssens, J., de Gucht, V., van der Doef, M., \& Maes, S. (2012). Exploring the burden of emergency care: predictors of stress-health outcomes in emergency nurses. Journal of Advanced Nursing, 67(6), 1317-1328. doi:10.1111/j.13652648.2010.05599.x

American Psychiatry Association (2013). DSM-5, Diagnostic and Statistical Manual of Mental Disorders ( $5^{\mathrm{a}}$ ed.). Washington, DC: Author.

Anakwenze, U., \& Zuberi, D. (2013). Mental Health and Poverty in Inner City. Health and Social Work, 38(3), 147-157. doi: 10.1093/hsw/hlt013

Araújo, T. M., \& Karasek, R. A. (2008). Validity and reliability of the job content questionnaire in formal and informal jobs in Brazil. Scandinavian Journal Work and Environmental Health Supplements, 6, 52-59.

Batista, A. G. (2009). Quando os bombeiros não chegam: algumas contribuições da Psicologia do Trabalho para o entendimento dos acidentes com veículos operacionais de bombeiros na Região Metropolitana de Belo Horizonte. (Unpublished master's thesis). Universidade Federal de Minas Gerais, Belo Horizonte. 
Berger, W., Coutinho, E. S. F., Figueira, I., Marques-Portella, C., Luz, M. P., Neylan, T. C.,... Mendlowicz, M. V. (2011). Rescuers at risk: a systematic review and meta-regression analysis of the worldwide current prevalence and correlates of PTSD in rescue workers. Social Psychiatry and Psychiatric Epidemiology, 47(6), 1001-1011. doi: 10.1007/s00127-0110408-2

Berger, W., Maurat, A. M., Bucassio, E. P., Vieira, I., Jardim, S. R., Coutinho, E. S. F.,...Mendlowicz, M. V. (2007). Partial and Full PTSD in Brazilian Ambulance Workers: Prevalence and Impact on Health and Quality of Life. Journal of Traumatic Stress, 20(4), 637-642. doi: 10.1002/jts.20242

Berger, W., Mendlowicz, M. V., Souza, W. F., \& Figueira, I. (2004). Equivalência semântica da versão em português da Post-Traumatic Stress Disorder Checklist - Civilian Version (PCL-C) para rastreamento do transtorno de estresse póstraumático. Revista de Psiquiatria do Rio Grande do Sul, 26(2), 167-175. doi: 10.1590/S0101-81082004000200006

Blein, L. M., Galovski, T. E., \& Robinson, T. (2010). Gender differences in recovery from posttraumatic stress disorder: A critical review. Aggression and Violent Behavior, 15:463-474. doi:10.1016/j.avb.2010.09.001

de Boer, J. C., van'tVerlaat, E., Duivenvoorden, H. J., Bakker, A. B., \& Smit, B. J. (2011). Work-related critical incidents in hospitalbased health care providers and the risk of post-traumatic stress symptoms, anxiety, and depression: a meta-analysis. Social Science \& Medicine, 73(2), 316-326. doi: 10.1016/j. socscimed.2011.05.009

Brewin, C. R., Andrews, B., \& Valentine, J. D. (2000). MetaAnalysis of Risk Factors for Posttraumatic Stress Disorder in Trauma-Exposed Adults. Journal of Consulting and Clinical Psychology, 68(5), 748-766.

Brewin, C. R., Lanius, R. A., Novac, A., Schnyder, U., \& Galea, S. (2009). Reformulating PTSD for DSM-V: Life After Criterion A. Journal of Traumatic Stress, 22(5), 366-373. doi: 10.1002/ jts. 20443

Carey, M. C., Al-Zaiti, S. S., Dean, G. E., Sessanna, L., \& Finnell, D. S. (2011). Sleep problems, depression, substance use, social bonding, and quality of life in professional firefighters. Journal of Occupational Environment \& Medicine, 53(8), 928-933. doi: 10.1097/JOM.0b013e318225898f

Chang, C. M., Lee, L.C., Connor, K. M., Davidson, J. R. T., \& Lai, T. J. (2008). Modification effects of coping on post-traumatic morbidity among earthquake rescuers. Psychiatry Research, 158(2), 164-171. doi: 10.1016/j.psychres.2006.07.015

Chau, N., Wild, P., Dehaene, D., Benamghar, L., Mur, J. M., \& Touron, C. (2010). Roles of age, length of service and job in work-related injury: a prospective study of 446120 personyears in railway workers. Occupational \& Environmental Medicine, 67(3), 147-153. doi: 10.1136/oem.2008.043281

Chen, Y. S., Chen, M. C., Chuou, F. H. C., Sun, F. C., Chen, P. C., Tsai, K. Y., \& Chao, S. S. (2007). The relationship between quality of life and posttraumatic stress disorder or major depression for firefighters in Kaohsiung, Taiwan. Quality of Life Research, 16(8), 1289-1297. doi: 10.1007/s11136-007-9248-7

Cyrulnik B. (2009) Autobiografia de um espantalho - histórias de resiliência: o retorno da vida. São Paulo: Editora WMF Martins Fontes.
Del Ben, K. S., Scotti, J. R., Chen, Y. C., \& Fortson, B. L. (2006). Prevalence of posttraumatic stress disorder symptoms in firefighters. Work \& Stress, 20(1), 37-48. doi: 10.1080/02678370600679512

Foa, E. B., Cashman, L., Jaycox, L., \& Perry, K. (1997). The validation of a self-report measure of PTSD: the PTSD Diagnostic Scale (PDS). Psychological Assessment, 9(4), 445-451.

Gold, S. D., Marx, B. P., Soler-Baillo, J. M., \& Sloan, D. M. (2005). Is life stress more traumatic than traumatic stress? Journal of Anxiety Disorders, 19(6), 687-698. doi: 10.1016/j. janxdis.2004.06.002

Hair, J. F., Anderson, R. E., Tatham, R. L., \& Black, W. (2005). Análise Multivariada de Dados. São Paulo: Artmed Editora S.A.

Heinrichs M., Wagner, D., Schoch, W., Soravia, L. M., Hellhammer, D. H., \& Ehlert, U. (2005). Predicting Posttraumatic Stress Symptons from Pretraumatic Risk Factors: A 2-Year Prospective Follow-Up Study in Firefighters. American Journal of Psychiatry, 162(12), 2276-2286. doi: 10.1176/appi. ajp.162.12.2276

Karasek, R. A., \& Theörell, T. (1990). Healthy Work - stress, productivity, and the reconstruction of working life. US: Basic Books.

Laposa, J. M., \& Alden, L. E. (2003). Posttraumatic stress disorder in the emergency room: exploration of a cognitive model. Behavior ResearchTherapy, 41(1), 49-65.

Lima, E. P., \& Assunção, A. A. (2011). Prevalência e fatores associados ao Transtorno de Estresse Pós-Traumático (TEPT) em profissionais de emergência: uma revisão sistemática da literatura. Revista Brasileira de Epidemiologia, 14(2), 217-230. doi: 10.1590/S1415-790X2011000200004

Lima, E. P., Barreto, S. M., \& Assunção, A. A. (2012). Factor structure, internal consistency and reliability of the Posttraumatic Stress Disorder Checklist (PCL): an exploratory study. Trends in Psychiatry and Psychotherapy, 34(4), 215-222. doi: 10.1590/ S2237-60892012000400007

Masur, J., \& Monteiro, M. G. (1983). Validation of the "CAGE" alcoholism screening test in a Brazilian psychiatric inpatient hospital setting. Brazilian Journal of Medicine and Biological Research, 16(3), 215-218.

Meyer, E. C., Zimering, R., Daly, E., Knight, J., Kamholz, B. W., \& Gulliver, S. B. (2012). Predictors of Posttraumatic Stress Disorder and Other Psychological Symptoms in TraumaExposed Firefighters. Psychological Services, 9(1), 1-15. doi: 10.1037/a0026414

Mitani, S. (2008). Comparative Analysis of the Japanese Version of the Revised Impact of Event Scale: a study of firefighters. Prehospital and Disaster Medicine, 23(3), 20-26.

Nieuwenhuijsen, K., Bruinvels, D., \& Frings-Dresen, M. (2010). Psychosocial work environment and stress-related disorders, a systematic review. Occupational Medicine, 60(4), 277-286. doi:10.1093/occmed/kqq081

Regehr, C., Millar, D. (2007) Situation critical: High demand, low control, and low support in paramedic organizations. Traumatology, 13(1), 49-58. doi: 10.1177/1534765607299912

Ozer E. J., Best, S. R., Lipsey, T. L., \& Weiss, D. S. (2003). Predictors of posttraumatic stress disorder and symptoms in adults: a meta-analysis. Psychological Bulletin, 129, 52-73. doi: 10.1037/1942-9681.S.1.3 
Rosen, G. M., \& Lilienfeld S. O. (2008). Posttraumatic Stress Disorder: an empirical evaluation of core assumptions. Clinical Psychology Review, 28(5), 837-868. doi: 10.1016/j. cpr.2007.12.002.

Silva, L. S., \& Barreto, S. M. (2010) Adverse psychosocial working conditions and minor psychiatric disorders among bank workers. BMC Public Health, 10, 686. doi: 10.1186/14712458-10-686

Slottje, P., Witteveen, A. B., Twisk, J. W. R., Smidt, N., Huizink, A. C., van Mechelen, W., \& Smid, T. (2008). Post-disaster physical symptons of firefighters and police officers: role of types of exposure and post-traumatic stress symptons. British Journal of Health Psychology, 13(2), 327-342. doi: 10.1348/135910707X198793

Su, T. P., Lien, T. C., Yang, C. Y., Su, Y. L., Wang, J. H., Tsai, S. L., \& Yin, J. C. (2007). Prevalence of psychiatric morbidity and psychological adaptation of the nurses in a structured SARS caring unit during outbreak: A prospective and periodic assessment study in Taiwan. Journal of Psychiatric Research, 41(1), 119-130. doi: 10.1016/j.jpsychires.2005.12.006

Tabachnick, B., \& Fidell, L. S. (1996). Using multivariate statistics (3a ed.). New York: Harper Collins
U'Ren, R. (2011) Social Perspective - The missing element in Mental Health Practice. Canada: University of Toronto Press. van der Velden, P. G., Christiaanse, B., Kleber, R. J., Marcelissen, F. G. H., Dorresteijn, S. A. M., Drogendijk, A. N.,... Meewisse, M. L. (2006). The effects of Disaster exposure and post-disaster critical incidents on intrusions, avoidance reactions and health problems among firefighters: a comparative study. Stress, Trauma and Crisis, 9(2) 73-93. doi:10.1080/15434610600683742

van der Velden, P. G., Kleber R. J., Grievink L., \& Yzermans J. C. (2010). Confrontations with aggression and mental health problems in police officers: the role of organizational stressors, life-events and previous mental health problems. Psychological Trauma (DNLM), 2(2), 135-144. doi: 10.1037/a0019158

Weathers, F. W., Litz, B. T., Herman, D. S., Huska, J. A., \& Keane, T. M. (1993, october). The PTSD Checklist (PCL): reliability, validity, and diagnostic utility. In Annual Meeting of International Society for Traumatic Stress Studies. San Antonio, TX.

World Health Organization (2010). Redução das desigualdades no periodo de uma geração. Igualdade na saúde através da acção sobre os seus determinantes sociais. Geneva: World Health Organization. 